\title{
Human Continuity through Crises in Aviation
}

\author{
Kimberly A. Kenville, James A. Higgins, Rosanne B. McBride, Thomas V. Petros, \\ Warren C. Jensen and Eleanor Yurkovich \\ University of North Dakota
}

\begin{abstract}
Highly-trained employees are essential to airports and aviation organizations. It is very important for managers as well as peers to recognize post traumatic stress reactions or symptoms that employees may display when they have attended to a natural or manmade disaster. This research project has identified and documented that many organizations practice the first-response activities associated with a traumatic event, but engage in little or no training for the recovery phase of the event/incident, which may last a long period of time. The research has identified many strategies which should edify and augment a mental health recovery plan (MHRP) so that an employees' natural resiliency is enhanced, or those that are unable to return to normal function can be identified and receive the necessary mental health attention. The entire research project was funded by the Airport Cooperative Research Program (ACRP) as part of the National Academies.
\end{abstract}

\section{INTRODUCTION}

This research project examines an aviation organization's ability to promote human resiliency and to provide guidance for those organizations to develop procedures and prepare for the impact of natural and manmade disasters they may face. The goal of this research is to prepare directors of airports and air carriers for the mental health recovery of employees, who have faced a traumatic event, and to promote and improve practices for enhancing employees' ability to successfully cope with such an event and build resilience.

\section{BACKGROUND}

According to the Federal Aviation Administration's (FAA) 2008-2012 Flight Plan, "our skies are safe," the industry has achieved an incredibly low rate of commercial (airline) fatal accidents (FAA, 2008a, p.1). In the past ten years this rate has dropped 57 percent. The FAA has implemented many new and enhanced safety initiatives in the past with the hope of achieving the lowest rate practical. The aviation industry has inherent risk associated with it, which means that accidents will occur, but presently they occur at a very low rate. The nation's airlines transport nearly two million passengers per day and employ nearly half a million workers (Air Transport Association, 2007).

Natural disasters disrupt thousands of lives each year and can do unimaginable damage in mere moments. Whether the disaster is fire, flood, hurricane, earthquake or tornado, the threat is immediate to human life, and the recovery process is usually long term. Recently, Hurricanes Katrina and Rita bore down on the southern United States engulfing the states of Louisiana and Mississippi, forest fires have greatly impacted the western United States, and tornadoes and floods have ravaged the Midwest.

The aviation industry is not immune to the effects of a natural disaster as the organizations (airports, airlines, and FBOs) involved may become instantly crippled, with effects felt throughout their local areas. However, airports and air transportation become a vital link to receiving needed supplies and restoring order by allowing disaster relief workers to begin their work. In the case of Hurricane Katrina, the New Orleans - Louis Armstrong International Airport was the staging point for all egress and ingress of the afflicted areas. The airport became the virtual lifeline to the people of southern Louisiana (Blanchard, 2008).

\section{LITERATURE REVIEW AVIATION REQUIREMENTS - DISASTER/EMERGENCY PLANNING}

Air Carriers (Part 121, 125 and 135)

The Federal Aviation Administration (FAA) currently requires all air carriers operating under 14 CFR 121, 125 or 135 to have 
established accident reporting procedures. These procedures must be published in the carrier's operations manual stipulated in 14 CFR 121.135, 125.73 and 135.23. Aside from this requirement, the FAA does not mandate any type of structured program dealing with issues of employee or operator resiliency after an accident; rather, the emphasis is keenly placed on an operator's ability to manage an acute emergency. 14 CFR 121.417 outlines the specific requirements needed by an air carrier in order to mitigate an actual emergency situation such as in-flight aircraft fires or hijackings, but does not list any post-event psychological or "trauma handling" regulations. Airlines that operate under 14 CFR 121 also have a regulatory requirement to assist family members of passengers cope with traumatic events under the 1996 Aviation Disaster and Family Assistance Act.

\section{Airports (GA and Part 139)}

In a review of the of the Airport Emergency Plan advisory circular (AC/150/5200-31B) currently in draft format, it appears the Federal Aviation Administration has initiated a number of changes for airports. This draft, if approved, will replace an advisory circular from 1999 . The substantial changes suggested in the new advisory circular primarily relate to the addition of National Fire Protection Association standards for equipment and training related to airport firefighters and the application of the National Incident Management System (NIMS) and Incident Command System (ICS).

Section 8 of the advisory circular, Airport Emergency Planning (AEP), outlines health and medical planning. It is evident that the advisory circular is oriented toward treatment, transport, and evacuation of injured persons, or the response actions; but, the plan does not address the actual airport workers' mental health issues that may arise from working during traumatic events. However, section 6-8-2 (6) does address potential utilization of mental health agencies; the circular indicates that an airport should ensure that the appropriate mental health services are available for disaster victims, survivors, bystanders, responders and their families, and other airport care-givers during response and recovery (FAA, 2008b, p. 82).
The FAA introduces the idea of Critical Incident Stress Management (CISM), but clearly leaves the concept and its implementation up to each individual airport. It is not evident whether the FAA will direct an airport to implement any sort of mental health programs for airport workers. It should be noted that the FAA's advisory circular on emergency planning pertains only to FAR Part 139 airports, which are those airports that serve regularly scheduled air carrier (FAR Part 121) operations with aircraft operating with more than nine seats on board. General aviation (GA) airports and other aviation organizations that do not have particular regulations guiding their operations are not required to have well-developed emergency plans. Therefore, it is important that those entities take time to develop plans that deal with the possibility of a traumatic event or incident.

\section{WHAT IS PSYCHOLOGICAL TRUAMA AND WHAT CAUSES IT?}

The physical and psychological response to any demand-positive or negative-is stress. Positive stress includes responses to events such as getting a promotion, getting married, or graduating from college. However, the term stress usually describes responses to negative demands such as taking a test, getting divorced, or performing under pressure. When faced with a source of negative stress, people must evaluate the situation, determine the realistic level of risk (and differentiating that from imagined or irrational perceptions of risk) and then evaluate how they are going to cope with the situation based on their own personal resources (e.g., physical strength, the ability to think clearly in a crisis, basic problem-solving abilities) and the potential for support from others (e.g., emotional support, access to necessary tangible resources; Lazarus, 1966; Lazarus \& Folkman, 1984).

The most extreme form of negative stress is traumatic stress-stress resulting from a traumatic event or situation. People experience traumatic stress in response to events such as natural disasters like earthquakes or hurricanes, motor vehicle collisions, physical or sexual assault/abuse, combat, industrial accidents, diagnosis of a life-threatening illness, lifethreatening medical situations like a heart attack, 
terrorist attacks, torture, or as in the present discussion, airline disasters (Noy, 2004).

During an actual traumatic event, this response is considered a normal, adaptive survival response to a situation that is perceived as life threatening. If an individual is able to establish safety by fighting or fleeing, it will often decrease, although not eliminate, the risk for long-term negative effects of the stressful event (Noy, 2004). However, traumatic events may not accommodate these survival responses, and individuals must attempt to cope with a situation that is perceived as life-threatening, uncontrollable, and/or inescapable-a situation that carries a higher risk for longer-term problems.

Life-threatening, inescapable situations can result in a different physical and psychological response-freezing or becoming immobilized. Although this response is less well understood from a physiological standpoint, it appears that the stress response activates a different part of the Autonomic Nervous System (ANS) that immobilizes the body and decreases the experience of pain or fear (e.g., people going limp and psychologically numb when being mauled by a bear) (Noy, 2004).

\section{POST TRAUMATIC STRESS AND HUMAN REACTIONS TO TRAUMA}

When an individual continues to experience a persistent traumatic stress reaction after the traumatic event has past, or post-trauma, it is called post-traumatic stress (American Psychiatric Association, 2000). Thus, a stress response that was adaptive and normal during a time a crisis becomes maladaptive when it persists after the traumatic event has passed. Post-traumatic stress is a human survival reaction or elements of this reaction that occur when there is no actual threat present- $a$ survival reaction that occurs at the wrong time. When post-traumatic stress is severe and persistent it is called Post-Traumatic Stress Disorder (PTSD) as described in the Diagnostic and Statistical Manual of Mental Disorders: Text Revision (DSM-TR)-the standard reference used for classifying and diagnosing psychiatric disorders (American Psychiatric Association, 2000).
According to the DSM-TR (American Psychiatric Association, 2000) diagnostic criteria, to qualify for a diagnosis of PTSD, one must have: (1) experienced an event that is life threatening or perceived as life threatening, (2) witnessed an event that is perceived as life threatening to others, or (3) heard about violence to or the unexpected or violent death of others. The latter can involve such things as watching a traumatic event unfold on television (e.g., Hurricane Katrina or the events of 9/11) or hearing about the death of a loved one-referred to as vicarious or secondary traumatization (Palm, Polusny \& Follette, 2004).

Further, one must exhibit persistent evidence (i.e., lasting more than one month) of: (1) persistent re-experiencing of the traumatic event (e.g., intrusive memories or thoughts, flashbacks, nightmares); (2) avoidance of reminders or the trauma that can involve physical avoidance or psychological "avoidance" or numbness in the form of dissociation; and (3) chronic hyper arousal of the autonomic nervous system (e.g., difficulties sleeping, problems concentrating, hyper vigilance, increased anxiety, exaggerated startle response).

One must also exhibit severe impairments in daily functioning (e.g., impaired relationships, employment problems) in addition to the criteria just described. Individuals for whom these same symptoms persist for less than one month would be classified as having Acute Stress Disorder (ASD; American Psychiatric Association, 2000). As noted previously, dissociation or removing oneself mentally from an inescapable situation is one possible response to traumatic stress. There is evidence that if dissociation is present in the early or acute stages of the traumatic stress reaction, the risk is increased for developing subsequent PTSD (Birmes, Brunet, Carreras, Ducasse, Charlet, Lauque, Sztulman \& Schmitt, 2003) although conflicting results have been reported (Wittman, Moergeli, \& Schnyder, 2006).

Symptoms of PTSD usually appear within the first three months following exposure to the traumatic event. However, a significant number of individuals may also experience delayedonset PTSD (Buckley, Blanchard, \& Hickling, 1996) in which symptoms may not appear for 
months or years (American Psychiatric Association, 2000). The duration of PTSD also varies. For trauma victims with early-onset PTSD, PTSD has been shown to persist from months to years following the disaster (Galea, Nandi, \& Vlahov, 2005). Even with appropriate treatment, PTSD can persist as a lifetime chronic condition with periods of exacerbation and remission of symptoms (Noy, 2004).

Much of the literature that addresses workplace critical incidents refers to manuals that provide procedures, support personnel, and guidance to manage the emergency (Federal Aviation Administration, 2008a). These publications greatly assist individuals who may not recall proper procedures or make an incorrect decision in the chaos of an emergency. With regard to personnel, publications and strategies are also available to provide guidance on critical incident stress management programs with the goal of improving resiliency and decreasing psychological trauma and its associated complications.

\section{BUSINESS CONTINUITY PLANNING}

It is commonly known that most businesses pay more attention to the practical matters of a potential business interruption than planning for the people side of the business, yet it is quite apparent that personnel are the most valuable asset to a company in times of distress (Nowlan, 2008). Therefore, "human continuity" is a crucial variable in disaster planning for any organization. Determining what and how to respond to the human or mental health issues that may be present after a traumatic event are extremely important.

Organizations need to recognize that there are several vulnerable stakeholder groups including staff, community, customers, suppliers and family members when trauma is present. It is advisable for companies to consider the wellbeing of all groups when attempting to return to normal operations. Both family and community members represent a tremendous source for recovery for employees, which can aid in the recovery process and reduce down time (Paton, 1999). According to Paton (1999), local government agencies might pursue this cost effective strategy of establishing goodwill and consider a similar course of action. This course of action should be considered as a comprehensive human resources (HR) continuity plan, which considers traumatic impacts for its staff. This HR plan could use vulnerability data to screen staff so that the organization identifies the demands of key staff and what effects of trauma they may experience as a result of the event (Paton, 1999).

As a disaster may render certain employees or employee groups incapable of performing their jobs, it is the role of managers and human resources representatives to understand this issue and find the appropriate support that is needed. Paton (1999) explains that recent thinking about support programs for staff is focusing on developing resilient organizational cultures. This would include "empowering staff and managers, and providing them with the knowledge, and skills to design and implement appropriate intrinsic risk-reducing and recovery strategies”. Due to the sheer magnitude of some events, this may prove to be a cost-effective strategy.

\section{METHODOLOGY AND DATA ANALYSIS}

The research team visited five different airports and qualitatively interviewed 25 individuals with varying work duties and training backgrounds, during this project. The research team also felt it would be valuable to try and determine which airports across the country had mental health components to their airport emergency plans (AEP) and whether or not those airports would be open to including such an area to their plan. Therefore, the team conducted an online survey of commercial service and general aviation airports.

\section{Online Airport Survey}

In order to determine the extent of postdisaster mental health crisis programs existing at airports in the United States, a survey was conducted among airport management personnel. A convenience sample was used from the membership roster of the American Association of Airport Executives (AAAE) 2008 National Conference attendees.

In general, representatives on the roster list were the highest ranking management official associated with a particular airport. Each member on the AAAE roster was sent an email 
which contained an internet link to an online survey instrument. This survey used the software on www.surveymonkey.com. To ensure anonymity, the survey did not require any participant to identify themselves or their Table 1. Online Survey Questions and Responses airports. The survey was administered to representatives of 175 airports nationwide and 64 responded, for a response rate of $37 \%$. The survey and its findings are listed in Table 1.

\begin{tabular}{|c|c|c|c|}
\hline Question & Response & Percent & $\mathrm{N}$ \\
\hline $\begin{array}{l}\text { In regard to your airport emergency plan } \\
\text { (AEP), does your airport currently have any } \\
\text { formal or informal program(s) designed to deal } \\
\text { exclusively with the mental health trauma that } \\
\text { employees may face after responding to aircraft } \\
\text { accident or natural disaster? }\end{array}$ & $\begin{array}{l}\text { Yes } \\
\text { No }\end{array}$ & $\begin{array}{l}56.3 \\
43.7\end{array}$ & $\begin{array}{l}35 \\
28\end{array}$ \\
\hline $\begin{array}{l}\text { If your organization has a mental health } \\
\text { component for your employees, please } \\
\text { describe. }\end{array}$ & $\begin{array}{l}\text { Multiple open- } \\
\text { ended responses }\end{array}$ & & \\
\hline $\begin{array}{l}\text { In the past } 10 \text { years, has your organization been } \\
\text { exposed to any types of disasters? (Check all } \\
\text { that apply). }\end{array}$ & $\begin{array}{l}\text { Airline } \\
\text { General Aviation } \\
\text { Natural Disaster }\end{array}$ & $\begin{array}{l}11.4 \\
68.6 \\
48.6\end{array}$ & $\begin{array}{c}4 \\
24 \\
17\end{array}$ \\
\hline $\begin{array}{l}\text { Would you be in favor of a program/template } \\
\text { that would help your organization initiate a } \\
\text { program to assist employees coping with } \\
\text { traumatic events (deal with what they have } \\
\text { witnessed) in the course of responding to a } \\
\text { disaster? }\end{array}$ & $\begin{array}{c}\text { Yes } \\
\text { No } \\
\text { Don’t Know }\end{array}$ & $\begin{array}{c}62.5 \\
9.4 \\
28.1\end{array}$ & $\begin{array}{c}40 \\
6 \\
18\end{array}$ \\
\hline
\end{tabular}

The airport locations were divided among Alaskan, Central, Eastern, Great Lakes, New England, Northwest Mountain, Southern, Southwest, and Western Pacific regions . Type of Airport (based upon FAA criterion) included general aviation, non hub, small hub, medium hub, and large hub. The yearly enplanements included the following choices: no enplanements, less than 100,000 enplanements, 100,001 to 250,000 enplanements, 250,001 to 500,000 enplanements, and over 500,000 enplanements. The survey had 64 respondents which covered all regions except Alaskan and all types of airports and enplanement categories. In addition, exposure to natural, airline and general aviation disasters within the preceding ten years was recorded.

The first question that respondents answered was the following: "In regard to your Airport Emergency Plan (AEP), does your airport currently have any formal or informal program(s) designed to deal exclusively with the mental health trauma that employees may face after responding to an aircraft accident or natural disaster?” Thirty-six respondents indicated they do currently have a program in place to deal with employee mental health traumas postaccident, while 28 indicated that they do not have such programs. It should be noted that no definition of a "formal or informal program" was used within the survey, and the interpretation was left up to the respondent. It is possible there are wide variances between the structures and types of programs amongst those answering in the affirmative.

Secondly, a question asked was the following: "Would you be in favor of a program/template that would help your organization initiate a program to assist employees coping with traumatic events (deal with what they have witnessed) in the course of responding to a disaster?” Forty respondents indicated they would be in favor, with six not in favor and 18 uncertain. There was no significant 
difference between those organizations who had post-disaster mental health trauma programs in place and those who did not with regard to being in favor of implementing such a program,

$$
\chi^{2}(2, N=64)=.666, p>.05 \text {. }
$$

Within the preceding 10 years, airports who had experienced an airline disaster $(\mathrm{n}=4)$, a general aviation disaster $(n=24)$, or a natural disaster $(n=17)$ reported no group differences in their preference for wanting post-disaster mental health programs, respectively.

$$
\chi^{2}(2, N=64)=4.693, p=.096 ; \chi^{2}(2, N=
$$

64) $=1.233, p>.05$; and, $\chi^{2}(2, N=64)=.205$, $p>.05$,

Airport location, classification and number of annual enplanements also demonstrated no group differences with regard to favoring or not favoring the creation of a program,

$$
\chi^{2}(14, N=64)=16.261, p>.05 ; \chi^{2}(8, N=
$$

$64)=5.908, p>.05$; and, $\chi^{2}(8, N=64)=4.388$, $p>.05$.

Interestingly, 36 of 64 (56\%) respondents reported they already have a mental health recovery program in place at their airport. This does not seem to be congruent with the larger study's findings in the field. These results could possibly be explained with a wide variance of definitions as they pertain to a mental health recovery programs. For instance, it is possible that an airport may simply have a clause in their emergency plan to have employees contact the Red Cross or the Employee Assistance Program (EAP) should they encounter mental health trauma. While this may be a productive measure, it may not be comprehensive enough to completely assist employees with their own resiliency and would not be considered a "classic" mental health recovery program.

The majority of respondents feel a mental health recovery program is a worthwhile addition to their plan (62.5\%). This perception held true irrespective of whether the airport already had a plan in place or not and whether they had experienced an aviation or natural disaster within the past 10 years. Only $9.4 \%$ did not favor the idea of such a program, with $28.1 \%$ unsure.

From the data, it appears most airports would be open to some type of guidance on how to implement a mental health recovery program and integrate it within their emergency plan. Regarding the favorability of implementing a program, extensive regulation or cumbersome application could be the reason for the higher number of "unsure" respondents. However, a formal definition of such a plan would have to be thoroughly developed and applied in order to alleviate burdensome obstacles in implementation. This definition could also increase the robustness of currently implemented plans, whether they are simplistic or involved. In any event, further study into the issue of mental health recovery programs would generate more focused data of a recovery program as they operationally defined.

\section{RECOMMENDATIONS}

In spite of the many defensive strategies and sound operating techniques employed, catastrophic aviation-related disasters occur. As any industry practitioner knows, it is vital to prepare for such events. Most preparation is aimed squarely on loss-of-life mitigation, scene preservation, and ultimately scenario reconstruction. However, an aspect that often gets overlooked involves the mental health monitoring, maintaining, and resilience of air carrier and airport employees. As with any critical incidence response, maintaining functional employee mental health is a vital component, and should be given due consideration prior to the occurrence of a catastrophic event.

Throughout the aviation industry, there are many different management structures in place at airports and air carriers. Delineating factors between such structures include size, resources and number of employees. Clearly, a large organization with several thousand employees will have different resources available than smaller operations with an employee or two. Irrespective of an organization's scope, there are several critical planning tasks the research team developed that should be common to all MHRPs and should be implemented as part of critical 
incident response plans. These tasks are defined below and outlined in figure 1 .

\section{Step 1. Awareness and Cultural Integration}

The first planning task of all organizations should simply be making all employees and any affected individuals aware that the organization will now be implementing a MHRP. Ideally, this should be stated in an employee manual or Airport Master Plan (AMP). The concept should be introduced and emphasized via several communication channels including verbal, signage and written policy. By engaging in such emphasis, the concept of an MHRP can become interwoven with the organization's culture. In addition, this emphasis may help alleviate (but probably not eliminate) some of the welldocumented phenomenon wherein some individuals are resistant to receiving mental health assistance.

\section{Step 2. Assessment of Mental Health Resource Availability}

In any disaster planning endeavor, it is critical to determine exactly what resources are available and which employees will be responsible for each of the necessary tasks. As previously discussed, most planning efforts focus on loss-of-life mitigation and scene preservation. As part of an MHRP, determining who will be responsible for overseeing the psychological-monitoring of the plan is equally important. Ideally, a licensed mental health practitioner who is employed by the organization would be the key person; however, it is very unlikely any organization would have the luxury of having such a person on staff.

However, almost all organizations have access to Employee Assistance Programs (EAPs). An EAP is a program in which employees have confidential access to mental health providers to help them through psychologically stressful events, like chemical dependence issues and traumatic personal events. Usually, these programs are accessed when an employee needs help and is willing to make first contact. In the case of implementing an MHRP, it is recommended that an EAP take more of a proactive status and actually seek out employees as part of the organizational team. Federal, state or locally governed organizations may be able to utilize a government sponsored
EAP (at least for the purposes of use during catastrophic events). Even if an organization does not currently have access to an EAP, it is highly recommended the organization contracts with some mental health entity for the purposes of implementing an employee MHRP during critical incidents.

\section{Step 3. Embedding Mental Health Practitioners}

Many current mental health monitoring programs in place make use of peer-to-peer sessions, often termed "debriefings" or "defusings." Without a doubt, sound operating practices dictate that logistical and progress briefings be made so as to ensure all personnel maintain the appropriate levels of awareness and situation status.

At issue is the possibility of an employee experiencing Post Traumatic Stress Disorder (PTSD). The current evidence indicates that unless an employee experiencing PTSD is assessed and treated by a licensed mental health provider, an untrained peer counselor could potentially exacerbate the stress levels (albeit unintentionally) of the employee and prolong the PTSD episode. It is important to note that some employees report they greatly desire a peer-topeer model, and believe such models have helped them in the past. However, the findings from the present study seem to belie this notion with some people and certainly demonstrate the requirement for more investigation into whether or not peer-based interventions should become the preferred treatment method.

In some cases, there is a stigma attached to seeking out professional mental health support from licensed providers. Given that the efficacy of peer-to-peer counseling is questionable at best, there seems to be a conundrum; how does an employer provide mental health assistance for their employees during a crisis when there is apprehension about seeking a professional and a peer may be unqualified to help? In order to overcome both obstacles, it is recommended that the employer embed licensed mental health professionals, preferably from the organization's EAP, as part of the internal team involved in a crisis. These professionals should literally "walk the scene" with all of the employees as everyone goes about their business of dealing 
with the catastrophe. Using this model as a component of a MHRP has proven to be effective and accepted by most employees as consistently demonstrated through the qualitative analysis of the project.

\section{Step 4. Preparations of the Mental Health Provider}

The embedded mental health provider should acquaint themselves with all of the available assessment and therapeutic techniques recognized as efficacious when treating PTSD or other associated trauma. A comprehensive and topical review of PTSD treatment for air carrier or airport crises can be found with the entire research document at the ACRP website http://www.trb.org/news/blurb_browse.asp?id=8 6.

\section{Step 5. Employee Training Program}

As part of the planning activities, all employees should be taught basic crisis management techniques and how to recognize PTSD symptoms. While peer-to-peer counseling should be limited, knowing how to recognize some symptoms in co-workers and advising the embedded mental health team member of such signs could prove helpful. In addition, a basic description of the cause, prognosis if left untreated, and long-term care principles regarding traumatic stress should be emphasized.

\section{Step 6. Establishment of a Mutual Aid Assistance Program}

Some airports participate in mutual aid groups whereby in the event of a natural crisis (hurricane, flood, etc.) other airports not affected will send personnel to staff critical functions. Certainly, this gives the ability for the airport to function; and, often airports are a vital asset during natural disaster recovery efforts. However, there is also a mental health component to participating in a mutual aid pact. Employees who work at an airport experiencing a natural disaster are often affected by the same disaster in their personal lives. They may be caught in a dilemma between continuing to work so as to support the airport's function or abandoning their posts so that they can deal with their own families and personal situations. By participating in a mutual aid group, an organization could help enable employees to deal with their personal situations and not make a difficult, stress-inducing decision between work and family.

This study identified two groups in existence at present. They are the Western Airports Disaster Operations Group (WESTDOG) and the South East Airports Disaster Operations Group (SEADOG). Contact with WESTDOG can be made through the Dallas-Ft. Worth International Airport (DFW) and contact with SEADOG can be done through Pensacola International Airport (PNS), Savannah/Hilton Head International Airport (SAV), or the Gulfport-Biloxi International Airport (GPT). Presently there are no known mutual aid programs between air carriers, and it is unlikely one could emerge due to competitive issues, operational complexities and regulatory oversight. However, intra-company mutual aid pacts should be considered between stations.

\section{Step 7. Assimilating the MHRP into Critical Incident Response Training}

The final step is to fully integrate MHRP concepts into any disaster/incident training undertaken by the organization. In the event of full-scale disaster simulations, the MHRP should also be simulated, practiced and evaluated so as to equip an organization with the necessary knowledge prior to an actual catastrophic event. As an example of such training, an organization could designate some employees to play a role of an overstressed employee by having that person exhibit certain symptoms that should be recognized by peers and evaluated by the embedded mental health provider.

The following figure outlines each of the steps for the planning phase of a MHRP. 


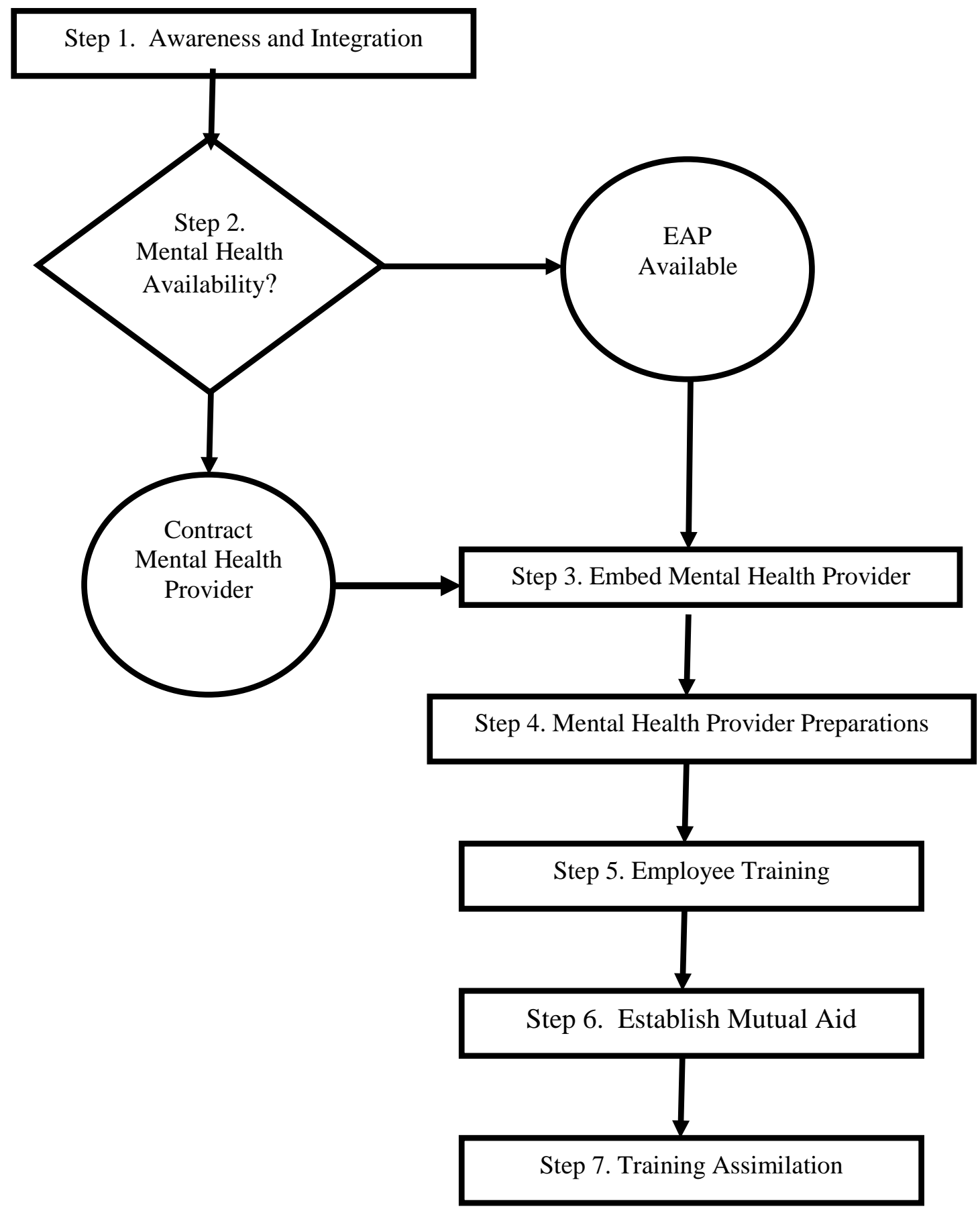

Figure 1. Mental Health Recovery Program Planning Steps 


\section{CONCLUSION}

Lastly, there is "no one size fits all" approach. Therefore careful consideration of an organization's employee perceptions and postevent mental health status, and the organizational structure, culture, and communication network is critical in framing an appropriate response to the traumatic event and realizing the best possible course of action for all involved. Many state and federal organizations do posses well-vetted MHRPs, it is paramount that each entity gathers all the needed information to carefully craft their individual plan and to assess how that plan will enhance their employee's resiliency in the face of trauma. 


\section{REFERENCES}

Air Transport Association (2007). Aviation Safety and other Priorities: A Statement of the Air Transport Association of America, Inc., before the Aviation Subcommittee of the House Transportation and Infrastructure Committee. March 22, 2007. Retrieved on June 24, 2008 from www.airlines.org

American Psychiatric Association (2000). Diagnostic and statistical manual for mental disorders (4 ${ }^{\text {th }}$ Ed., Text Revision). Washington D.C.: Author.

Birmes, P., Brunet, A., Carreras, D., Ducasse, J., Charlet, J., Lauque, D., Sztulman, H., \& Schmitt, L. (2003). The predictive power of peritraumatic dissociation and acute stress symptoms for posttraumatic stress symptoms: A three-month prospective study. American Journal of Psychiatry, $160,1337-1339$.

Blanchard, K. (2008). Airport Planning for natural disasters. American Association of Airport Executives (AAAE) Annual Conference. June 8, 2008. (Retired Fire Chief, New Orleans - Louis Armstrong International Airport).

Buckley, T.C., Blanchard, E.B, \& Hickling, E.J. (1996). A prospective examination of delayed-onset PTSD secondary to motor vehicle accidents. Journal of Abnormal Psychology, 103:617-625.

Code of Federal Regulations 14 CFR Part(s) 121, 135, 139, Federal Aviation Regulations. http://ecfr.gpoaccess.gov/cgi/t/text/textidx?sid=9083a033d658c5ecde5dcc4a61cf1c8b\&c=ecfr\&tpl=/ecfrbrowse/Title14/14tab_02.tpl

Code of Federal Regulations 49 CFR Part 830, Federal Aviation Regulations / Aeronautical Information Manual (FAR/AIM), ASA, 2005.

Department of Homeland Security. (2004). National Incident Management System. Retrieved on June 25, 2008 from http://www.nimsonline.com/docs/NIMS-90-web.pdf

Disaster Mental Health for Responders: Key Principles, Issues and Questions, Center for Disease Control website, www.bt.cdc.gov/mentalhealth/responders.asp

Federal Aviation Administration (2008a). 2008-2012 FAA Flight Plan: Charting the Path for the Next Generation. Retrieved on June 24, 2008 at www.faa.gov

Federal Aviation Administration (2008b). Airport emergency planning. Advisory circular 150/5200-31B. draft. Department of Transportation.

Federal Bureau of Investigation (FBI). (2005). Victim Assistance. Retrieved on July 7, 2008 from http://www.fbi.gov/hq/cid/victimassist/resources.htm

Federal Family Assistance Plan, National Transportation Safety Board, 2000.

Galea, S., Nandi, A., \& Vlahov, D. (2005). The Epidemiology of Post-Traumatic Stress Disorder after Disasters. Epidemiologic Reviews, 27, 78-91.

Lazarus, R.S. (1966). Psychological stress and coping processes. New York, NY: McGraw Hill.

Lazarus, R.S. \& Folkman, S. (1984). Stress, appraisal, and coping. New York, NY: Springer.

National Response Team (NRT). (n.d.). Incident Command System (ICS)/Unified Command (UC). Technical Assistance Document. Retrieved on July 1, 2008 from: http://www.nrt.org/Production/ NRT/NRTWeb.nsf/AllAttachmentsByTitle/SA-52ICSUCTA/\$File/ICSUCTA.pdf?OpenElement

Nowlan, K. (2008). Business continuity. Strategic HR Review. Vol 7:3. P 41-42. Retrieved on September 16, 2008 from http://web.ebscohost.com/ehost/detail?vid=7\&hid=109\&sid=e9772bf9-85b7-4076$80 \mathrm{fb}-4 \mathrm{e}$ 
Noy, S. (2004). The traumatic process: conceptualization and treatment. Prehospital and Disaster Medicine, 19(1):37-45.

Palm, K.M., Polusny, M.A., \& Follette, V.M. (2004). Vicarious traumatization: Potential hazards and interventions for disaster and trauma workers. Pre-hospital and Disaster Medicine, 19(1):73-78.

Paton, D. (1999). Disaster business continuity: promoting staff capability. Journal of Disaster Prevention and Management. vol 8:2. Pp. 127-133.

Wittman, L., Moergeli, H., \& Schnyder, U. (2006). Low predictive power of peritraumatic dissociation for PTSD symptoms in accident survivors. Journal of Traumatic Stress, 19(5), 639-651.

World Health Organization (WHO). (2006). WHO IASC guidelines on mental health and psychosocial support in emergency settings. http://www.who.int/hac/network/interagency/news/ iasc_guidelines_mental_health_psychososial_text.pdf 